\title{
A Century of Economic Policy Uncertainty Through the French-Canadian Lens ${ }^{\star}$
}

\author{
David Ardia $^{\mathrm{a}, *}$, Keven Bluteau ${ }^{\mathrm{a}, \mathrm{b}}$, Alaa Kassem ${ }^{\mathrm{a}}$ \\ ${ }^{a}$ Department of Decision Sciences, HEC Montréal, Montréal, Canada \\ ${ }^{b}$ Department of Economics, Ghent University, Belgium
}

Economics Letters, 2021, volume 205, 109938

https://doi.org/10.1016/j. econlet. 2021. 109938

\begin{abstract}
A novel token-distance-based triple approach is proposed for identifying EPU mentions in textual documents. The method is applied to a corpus of French-language news to construct a century-long historical EPU index for the Canadian province of Quebec. The relevance of the index is shown in a macroeconomic nowcasting experiment.
\end{abstract}

Keywords: Economic uncertainty, Policy uncertainty, Canada, Quebec, token-distance-based triple, issues, nowcasting, Sentometrics

JEL: C43, C53, D8, E60

\section{Introduction}

The Economic Policy Uncertainty (EPU) index developed by Baker et al. (2016) is an important indicator in economic and financial applications (see, e.g., Caldara et al., 2019; Aliaga-Díaz et al., 2019; Brogaard and Detzel, 2012; Chen et al., 2016; Sum, 2012). Following the seminal American EPU index, several country/region-specific indices have been developed (see, e.g., Algaba et al., 2020; Ghirelli et al., 2019; Donadelli et al., 2020), including a Canadian index that uses articles from five English-language Canadian newspapers and starts in 1985. This note uses a unique French Canadian news corpus to construct

\footnotetext{
${ }^{\star}$ We are grateful to the Bibliothèque et Archives Nationales du Québec via the CO.SHS Corpus BAnQ initiative (https://co-shs.ca/en), the Canada Foundation for Innovation (https://www.innovation. ca), and CBC/Radio-Canada (https://cbc.radio-canada.ca) for providing us with the data and to IVADO and the Swiss National Science Foundation (grants \#179281 and \#191730) for their financial support. A special thanks to Stéphane Caron and Dany Plourde for making this project possible. We also thank the Editor (Eric Young), an anonymous referee, Andres Algaba, Noemi Ardia, Scott R. Baker, Clément Aymard, Kris Boudt and Carlos Ordas for their comments and Andres Algaba, Samuel Borms, Kris Boudt and Jeroen van Pelt for providing us with their French EPU lexicon. We acknowledge Noemi Ardia and Michel Veilleux for their unconditional support.

${ }^{*}$ Corresponding author. HEC Montréal, 3000 Chemin de la Côte-Sainte-Catherine, Montreal, QC H3T 2A7. Phone: +1 5143406103 .

Email addresses: david.ardia@hec.ca (David Ardia), keven.bluteau@hec.ca (Keven Bluteau), alaa.kassem@hec.ca (Alaa Kassem)
} 
more than a century of Canadian EPU from the province of Quebec's perspective. Quebec is a key player in the Canadian economy; it has more than $20 \%$ of the Canadian population and is the second contributor to Canadian GDP after Ontario. About 80\% of Quebec residents use French as their main language.

Our data set and the historical aspect of our index raise some challenges. First, the archives of several news sources are grouped by issue rather than by individual articles, which renders the standard EPU count impossible. Second, the availability of archives for particular media sources differs over time, making the static-window standardization used in the traditional sources' aggregation biased.

We propose a novel token-distance-based triple approach to identify EPU mentions in textual documents and use a dynamic normalization for the sources' aggregation to address these problems. We believe our methodology will help researchers compute EPU-like measures from a wider range of textual sources (both in terms of variety and availability) in future studies. We show that our EPU index spikes at major regional and worldwide events. Finally, we show that our index outperforms the existing Canadian EPU when nowcasting major Canadian and Quebec macroeconomic variables.

\section{Data and methodology}

\subsection{Data}

We consider four sources of French-language news available in Quebec. Archives for three newspapers are retrieved from the Bibliothèque et Archives Nationales du Québec (BAnQ): (i) La Presse (January 1928 - December 2013), (ii) Le Devoir (January 1910 December 2011) and (iii) Le Soleil (January 1972 - December 2006). The original daily issues for these three sources are converted into a computer-readable format using optical character recognition (OCR) technology. The fourth source consists of French-language news articles from Radio-Canada (the francophone arm of the Canadian Broadcasting Corporation) from January 2003 to June 2020. We process the raw data set the standard way. We transform our textual documents (issues and articles) into vectors of tokens using the Word Boundary Rules defined in the Unicode ${ }^{\circledR}$ Standard. ${ }^{12}$

\subsection{Index construction methodology}

Our index construction deviates from Baker et al. (2016) in three aspects: (i) dictionary, (iii) EPU count and (iii) source aggregation.

\footnotetext{
${ }^{1}$ Tokens are the individual units of texts; see http://www.unicode.org/reports/tr29/\#Word_ Boundaries. We remove any tokens that are French stop words (e.g., "le"), punctuation, and that contain numeric or non-alphanumeric characters.

${ }^{2}$ To illustrate our text processing, the sentence "Le salaire minimum passera à 13,50\$ l'heure samedi.", is represented by the five-token vector [salaire, minimum, passera, heure, samedi] at the end of the process. More details is available in the Internet appendix.
} 
Dictionary. As we are examining French documents, we rely on a list of French EPU keywords instead of the original list of English keywords. We use the dictionary provided by Algaba et al. (2020), which is based on a translation-enhanced word2vec approach, and add a few tax-related words specific to Canada or Quebec. ${ }^{3}$

$E P U$ count. For three of our sources, news is aggregated by issue. It is impossible to identify individual articles in an automated way, so we cannot perform the standard article-based EPU count. Instead, we try to identify EPU mentions by measuring the distance between tokens in EPU triples contained in each document. First, we determine the token positions of all EPU keywords occurring in the text. From these identified keywords, we construct all possible EPU triples for which we measure the maximum relative distance between the token-triple positions (i.e., the maximum of the absolute difference between the token position of the E-P, E-U and P-U keywords). ${ }^{4}$ We only keep triples with a maximum distance less than or equal to a given threshold $\tau$. The threshold aims to avoid mixing EPU triples between unrelated articles within an issue (i.e., it controls for false positives). ${ }^{5}$ From this subset, to avoid multiple counting, we count the number of unique triples: $\operatorname{Tr}_{d, t, s}$ denotes the count in document $d$ published in month $t$ by source $s .{ }^{6}$ Then, following Baker et al. (2016), we aggregate and scale our counts by source each month to get a monthly source-specific frequency-count measure:

$$
F_{t, s}=\frac{\sum_{d=1}^{D_{t, s}} \operatorname{Tr} i_{d, t, s}}{\sum_{d=1}^{D_{t, s}} \operatorname{Tok}_{d, t, s}},
$$

where $T o k_{d, t, s}$ denotes the total number of tokens for document $d$ published in month $t$ by source $s$, and $D_{t, s}$ denotes the total number of documents published in month $t$ by source $s$.

Source aggregation. In Baker et al. (2016), the source-specific frequency-count variables $F_{t, s}$ are first standardized by their standard deviation computed over a fixed and common time period. They are then aggregated to build the EPU index. ${ }^{7}$ The standardization aims to account for differences in the variability of sources' reporting. However, the availability

\footnotetext{
${ }^{3}$ We also test several enhancements of the dictionary using Latent Dirichlet Allocation on the news selected at EPU spikes over various periods. Results with alternative lexicons are qualitatively similar.

${ }^{4}$ If a keyword comprises more than one token, leading to multiple token positions for the keyword and thus multiple possible distances for a given triple, the minimum distance is taken.

${ }^{5}$ We use $\tau=125$ tokens, the median number of tokens in Radio-Canada articles. For Radio-Canada, we set $\tau=\infty$ as this source is available at the article level. We also test alternative setups, and find that results are similar for values ranging from $\tau=50$ to $\tau=1,000$. Lower values are too restrictive, and larger values result in triples being observed in unrelated news articles, leading to a noisier index.

${ }^{6} \mathrm{~A}$ unique triple is defined as a set of three unique token positions. For instance, consider two triples with positions $\{1,4,8\}$ and $\{1,7,10\}$. They count as a unique triple since position 1 appears in both. In other words, a token position can only be used once.

${ }^{7}$ The fixed-time window ranges from 1985 to 2009 for the American EPU, while the Canadian EPU relies on data before 2011.
} 
of our sources differs across time periods. Hence, the variability but also the level of $F_{t, s}$ are period-specific, making the traditional aggregation biased. ${ }^{8}$ To tackle this, we propose a dynamic approach. In month $t$, we first scale $F_{t, s}$ by its $m$-past months (rolling-window) standard deviation to ensure similar variation across sources. Second, we divide the scaled variable by its $m$-past months average to ensure similar levels across sources. ${ }^{9}$ Then, we aggregate the source-specific scaled and normalized frequency-count measures, $E P U_{t, s}$, to construct the EPU index in month $t$ :

$$
E P U_{t}=\sum_{s=1}^{S_{t}} \frac{E P U_{t, s}}{S_{t}}
$$

where $S_{t}$ denotes available sources in month $t$.

Our EPU construction ensures the index in month $t$ is only based on data available up to month $t$ (i.e., it is not forward-looking biased), which can be critical for practical applications such as forecasting. Note that the length of the rolling-window $m$ makes the interpretation of the EPU relative to its past $m$-month values. ${ }^{10}$ We believe it is a reasonable approach compared to selecting a single fixed reference period, especially for such a long time frame. The dynamic normalization accounts for a possible evolution in the media's writing style and type of news coverage. ${ }^{11}$

\section{Validation of the index}

In Figure 1, we display the evolution of our EPU index (January 1913 to June 2020) together with the historical American EPU (January 1900 to October 2014) and the Canadian EPU (January 1985 to June 2020). ${ }^{12}$ We see that our index spikes at major economic events. When looking over our 100-year time horizon, three peaks are particularly large: (i) the Great Depression, (ii) the 2008 financial crisis, and (iii) the COVID-19 pandemic. Moreover, the index tends to be higher post-war and during some other events such as the patriation of the Canadian constitution in 1982 (a national event) or the Oka crisis in 1990 (a Quebec-specific event). In comparison, the American index does not spike during the Great Depression or the post-war recession, raising questions about its accu-

\footnotetext{
${ }^{8}$ For instance, consider two sources whose EPU count frequency levels are different (one high and one low). If the low-count source disappears from the sample, the aggregate EPU will increase, despite the absence of an EPU event. We refer to the Internet appendix for illustrations.

${ }^{9}$ We set $m=36$ months (i.e., three-year rolling windows). An alternative setup with $m=60$ leads to similar graphical results with a correlation of 0.94 , and nonsignificant different nowcasting performance; see the Internet appendix.

${ }^{10}$ We set $m=36$ months in our empirical applications. Results with $m=60$ are qualitatively similar and are available in the Internet appendix.

${ }^{11}$ In the Internet appendix, we show that the dynamic approach better identifies historical events/crises and provides better nowcasting performance than a fixed-window approach as in Baker et al. (2016).

${ }^{12}$ The American and Canadian EPU indices are available at https ://www. policyuncertainty.com.
} 
racy. ${ }^{13}$ The Canadian EPU seems to track the Quebec EPU until 2010, when we start to see a large discrepancy whereby the Canadian EPU increases with no economic rationale. This discrepancy could be attributed to differences in calculation methodologies and/or data sources used to build the indices. A change in the media landscape could also explain this upward trend in the Canadian EPU, justifying the need for a dynamic normalization approach.

[Insert Figure 1 about here.]

\section{Nowcasting macroeconomic variables}

We now investigate our EPU index's effectiveness in nowcasting five monthly macroeconomic variables: (i) log-changes in Canadian gross domestic product (GDP), (ii) logchanges in Canadian and Quebec consumer price indices (CPIs), and (iii) changes in Canadian and Quebec unemployment rates (UNEMPs). ${ }^{14}$ We use the following specification:

$$
\mathcal{M}_{0}: \quad y_{t \mid t+1}=\beta_{0}+\beta_{1} y_{t-1 \mid t}+\beta_{2} E P U_{t \mid t}+\boldsymbol{\theta}^{\prime} \mathbf{x}_{t-1 \mid t}+\epsilon_{t},
$$

where $y_{t \mid \bullet}$ is the macroeconomic variable of interest, $\mathbf{x}_{t-1 \mid \bullet}$ is a vector of lagged explanatory variables, and $\epsilon_{t}$ is an error term. We consider a large set of Canadian and Quebec macroeconomic variables in $\mathbf{x}_{t-1 \mid \bullet}$; see Fortin-Gagnon et al. (2018). ${ }^{15}$ With our conditioning notation, we emphasize that all variables but the EPU are available with a time lag. We aim to evaluate out-of-sample nowcasting performance estimating the model on 60-month rolling windows starting in January 1985 and ending in June 2020. ${ }^{16}$ This, however, results in a smaller sample size than the number of coefficients needed to be estimated, making the ordinary least squares framework unfeasible.

To deal with the high dimension, we proceed in two ways (see Ardia et al., 2019). First, we estimate the model with an elastic-net (Zou and Hastie, 2005). Elastic-net is a penalized linear regression model that performs variable selection and shrinks regression coefficients towards zero, making the estimation of a model where the number of coefficients is larger than the number of observations feasible. The extent of the penalization is driven by hyper-parameters, which we select using the BIC-like criterion of Zou et al. (2007). Second, we reduce the dimensionality of the explanatory variables using principal components. This transformation into a lower-dimensional space then allows estimating the model using ordinary least squares. We select the number of optimal principal components following Bai and $\mathrm{Ng}$ (2002).

\footnotetext{
${ }^{13}$ According to https : //www.policyuncertainty.com, the American historical index is still a work in progress.

${ }^{14}$ Quebec GDP is not available at the monthly frequency.

${ }^{15}$ The full dataset is available at https://www.stevanovic.uqam.ca/DS_LCMD.html. In our nowcasting exercise, the number of available variables ranges from 130 to 140 .

${ }^{16}$ We choose this time window to compare our EPU with the Canadian EPU.
} 
Performance results are reported in Table 1 with our baseline model $\mathcal{M}_{0}$ together with a model $\mathcal{M}_{1}$ without EPU (i.e., $\beta_{2}=0$ ) and a model $\mathcal{M}_{2}$ for which the EPU is the Canadian EPU. First, for both the elastic-net and the principal components approaches, we find that our EPU-enhanced model has better results than the model using only macroeconomic variables $\left(\mathcal{M}_{0}\right.$ vs. $\left.\mathcal{M}_{1}\right)$. This holds for the root-mean-square forecast error (RMSFE) and the mean absolute forecast error (MAFE) performance metrics. The improvement is statistically significant at the $5 \%$ level for Canadian GDP and for Canadian and Quebec unemployment. Hence, our Quebec EPU helps to nowcast macroeconomic variables at the provincial and national levels. Next, we find that our EPU outperforms the nowcasting ability of the traditional Canadian $\operatorname{EPU}\left(\mathcal{M}_{0}\right.$ vs. $\left.\mathcal{M}_{2}\right)$. For both the estimation methods or the performance metrics, the outperformance is significant at the $5 \%$ level for Canadian GDP and unemployment rates for Quebec and Canada. ${ }^{17}$

[Insert Table 1 about here.]

\section{A. Internet appendix}

The Internet appendix contains additional information about the database, methodology and nowcasting performance of the models. The EPU index is available at https:// sentometrics-research.com.

\section{References}

Algaba, A., Borms, S., Boudt, K., Pelt, J.V., 2020. The economic policy uncertainty index for Flanders, Wallonia and Belgium. BFW digitaal / RBF numérique 6.

Aliaga-Díaz, R., Patterson, A., Raithatha, S., Hirt, J., Maciulis, V., 2019. Know unknowns : Uncertainy, volatility, and the odds of recession. Technical Report. Vanguard.

Ardia, D., Bluteau, K., Boudt, K., 2019. Questioning the news about economic growth: Sparse forecasting using thousands of news-based sentiment values. International Journal of Forecasting 35, 1370-1386. doi:10.1016/j.ijforecast.2018.10.010.

Bai, J., Ng, S., 2002. Determining the number of factors in approximate factor models. Econometrica 70, 191-221. doi:10.1111/1468-0262.00273.

Baker, S.R., Bloom, N., Davis, S.J., 2016. Measuring economic policy uncertainty. The Quarterly Journal of Economics 131, 1593-1636. doi:10.1093/qje/qjw024.

Brogaard, J., Detzel, A.L., 2012. The asset pricing implications of government economic policy uncertainty. doi:10.2139/ssrn.2105786. Working paper.

Caldara, D., Iacoviello, M., Molligo, P., Prestipino, A., Raffo, A., 2019. Does trade policy uncertainty affect global economic activity? Technical Report. The FED.

Chen, J., Jiang, F., Tong, G., 2016. Economic policy uncertainty in China and stock market expected returns. doi:10.2139/ssrn.2808862. Working paper.

\footnotetext{
${ }^{17}$ Additional analyses reported in the Internet appendix show that similar nowcasting results are obtained with alternative rolling-window lengths, while a static-window approach deteriorates the performance.
} 
Donadelli, M., Gufler, I., Pellizzari, P., 2020. The macro and asset pricing implications of rising Italian uncertainty: Evidence from a novel news-based macroeconomic policy uncertainty index. Economics Letters 197, 109606. doi:10.1016/j . econlet.2020.109606.

Fortin-Gagnon, O., Leroux, M., Stevanovic, D., Surprenant, S., 2018. A large Canadian database for macroeconomic analysis. Technical Report. CIRANO.

Ghirelli, C., Pérez, J.J., Urtasun, A., 2019. A new economic policy uncertainty index for Spain. Economics Letters 182, 64-67. doi:10.1016/j.econlet.2019.05.021.

Sum, V., 2012. The impulse response function of economic policy uncertainty and stock market returns: A look at the Eurozone. doi:10.2139/ssrn.2088700. Working paper.

Zou, H., Hastie, T., 2005. Regularization and variable selection via the elastic net. Journal of the Royal Statistical Society: Series B 67, 301-320. doi:10.1111/j.1467-9868.2005.00503.x.

Zou, H., Hastie, T., Tibshirani, R., 2007. On the "degrees of freedom" of the lasso. The Annals of Statistics 35, 2173-2192. doi:10.1214/009053607000000127. 


\section{Table 1: Nowcasting results}

The table reports the root-mean-square forecast error (RMSFE) and the mean absolute forecast error (MAFE) for the various models applied to five monthly macroeconomic variables: Canadian gross domestic product (GDP, log-changes), consumer price index (CPI, log-changes), and unemployment rate (UNEMP, changes); and Quebec CPI and UNEMP. We consider $\mathcal{M}_{0}$ in (3) and two alternatives: $\mathcal{M}_{1}$ is without EPU (i.e., $\beta=0$ ) and $\mathcal{M}_{2}$ is with the Canadian EPU instead of our EPU. Squared parentheses report the p-value of a Diebold-Marino test of

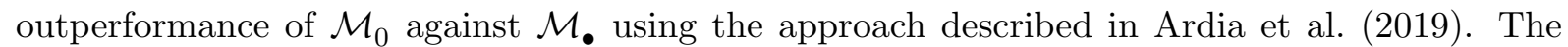
out-of-sample performance window ranges from January 1990 to June 2020 for a total of 366 observations (the first rolling window used to estimate the models ranges from January 1985 to December 1989).

\begin{tabular}{lccccccc}
\hline \multirow{2}{*}{ Panel A: Regularization (elastic-net) } & & \multicolumn{3}{c}{ MAFE (x100) } \\
\cline { 2 - 4 } \cline { 6 - 8 } Variable & $\mathcal{M}_{0}$ & $\mathcal{M}_{1}$ & $\mathcal{M}_{2}$ & & $\mathcal{M}_{0}$ & $\mathcal{M}_{1}$ & $\mathcal{M}_{2}$ \\
\hline GDP (CAN) & 1.041 & $1.415[0.000]$ & $1.429[0.002]$ & & 0.341 & $0.383[0.002]$ & $0.384[0.096]$ \\
CPI (CAN) & 0.369 & $0.367[0.828]$ & $0.379[0.038]$ & & 0.272 & $0.272[0.613]$ & $0.282[0.043]$ \\
UNEMP (CAN) & 0.441 & $0.604[0.000]$ & $0.608[0.000]$ & & 0.177 & $0.193[0.001]$ & $0.194[0.000]$ \\
CPI (QC) & 0.425 & $0.425[0.585]$ & $0.440[0.016]$ & & 0.299 & $0.302[0.415]$ & $0.316[0.075]$ \\
UNEMP (QC) & 0.989 & $1.254[0.000]$ & $1.264[0.000]$ & & 0.334 & $0.353[0.001]$ & $0.358[0.000]$
\end{tabular}

Panel B: Principal components

\begin{tabular}{lccccccc} 
& \multicolumn{3}{c}{$\operatorname{RMSFE}(\mathrm{x} 100)$} & & \multicolumn{3}{c}{ MAFE (x100) } \\
\cline { 2 - 5 } \cline { 6 - 8 } Variable & $\mathcal{M}_{0}$ & $\mathcal{M}_{1}$ & $\mathcal{M}_{2}$ & & $\mathcal{M}_{0}$ & $\mathcal{M}_{1}$ & $\mathcal{M}_{2}$ \\
\hline GDP (CAN) & 1.139 & $1.506[0.000]$ & $1.479[0.000]$ & & 0.349 & $0.382[0.000]$ & $0.380[0.000]$ \\
CPI (CAN) & 0.371 & $0.377[0.101]$ & $0.382[0.026]$ & & 0.273 & $0.277[0.205]$ & $0.280[0.030]$ \\
UNEMP (CAN) & 0.435 & $0.591[0.001]$ & $0.584[0.000]$ & & 0.171 & $0.186[0.000]$ & $0.185[0.000]$ \\
CPI (QC) & 0.431 & $0.433[0.364]$ & $0.439[0.143]$ & & 0.306 & $0.308[0.354]$ & $0.316[0.062]$ \\
UNEMP (QC) & 1.017 & $1.250[0.000]$ & $1.241[0.000]$ & & 0.336 & $0.342[0.003]$ & $0.347[0.000]$ \\
\hline
\end{tabular}




\section{Figure 1: EPU indices and events}

This figure displays our EPU index (solid black line, from January 1913 to June 2020 for a total of 1,290 observations) together with the Canadian EPU index (solid gray line, from January 1985 to May 2020 for a total of 425 observations) and the historical American EPU index (dotted line, from January 1900 to October 2014, for a total of 1,378 observations).

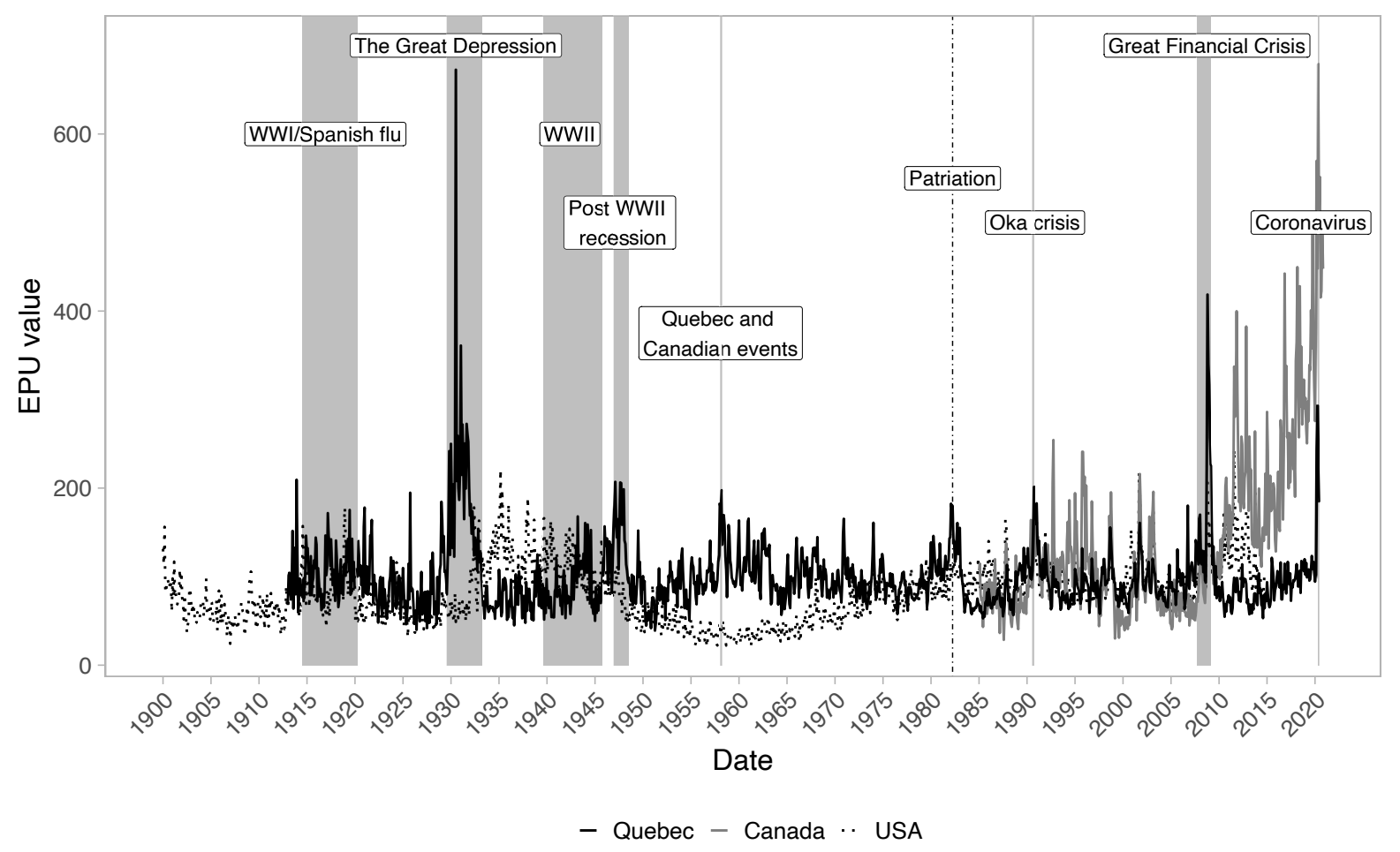

